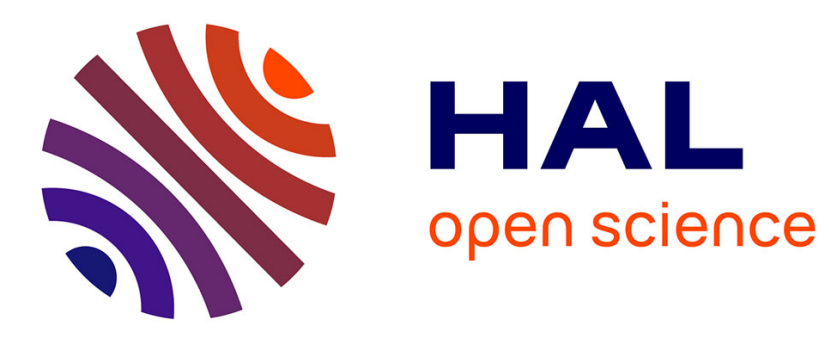

\title{
A spherical convolutional neural network for white matter structure imaging via dMRI
}

Sara Sedlar, Abib Alimi, Théodore Papadopoulo, Rachid Deriche, Samuel Deslauriers-Gauthier

\section{- To cite this version:}

Sara Sedlar, Abib Alimi, Théodore Papadopoulo, Rachid Deriche, Samuel Deslauriers-Gauthier. A spherical convolutional neural network for white matter structure imaging via dMRI. MICCAI 2021 - 24th International Conference on Medical Image Computing and Computer Assisted Intervention, Sep 2021, Strasbourg / Virtual, France. pp.Pages 529-539, 10.1007/978-3-030-87199-4_50 . hal03307031

\section{HAL Id: hal-03307031 \\ https://hal.science/hal-03307031}

Submitted on 29 Jul 2021

HAL is a multi-disciplinary open access archive for the deposit and dissemination of scientific research documents, whether they are published or not. The documents may come from teaching and research institutions in France or abroad, or from public or private research centers.
L'archive ouverte pluridisciplinaire HAL, est destinée au dépôt et à la diffusion de documents scientifiques de niveau recherche, publiés ou non, émanant des établissements d'enseignement et de recherche français ou étrangers, des laboratoires publics ou privés. 


\title{
A spherical convolutional neural network for white matter structure imaging via dMRI
}

\author{
Sara Sedlar, Abib Alimi, Théodore Papadopoulo, Rachid Deriche, and Samuel \\ Deslauriers-Gauthier \\ Inria, Université Côte d'Azur, FR \\ ffirstname.lastname\}@inria.fr
}

\begin{abstract}
Diffusion Magnetic Resonance Imaging (dMRI) is a powerful non-invasive and in-vivo imaging modality for probing brain white matter structure. Convolutional neural networks (CNNs) have been shown to be a powerful tool for many computer vision problems where the signals are acquired on a regular grid and where translational invariance is important. However, as we are considering dMRI signals that are acquired on a sphere, rotational invariance, rather than translational, is desired. In this work, we propose a spherical CNN model with fully spectral domain convolutional and non-linear layers. It provides rotational invariance and is adapted to the real nature of dMRI signals and uniform random distribution of sampling points. The proposed model is positively evaluated on the problem of estimation of neurite orientation dispersion and density imaging (NODDI) parameters on the data from Human Connectome Project $(\mathrm{HCP})$.
\end{abstract}

Keywords: Spherical CNN · diffusion MRI · white matter micro-structures

\section{Introduction}

Diffusion MRI (dMRI) is an imaging modality that exploits the interactions of diffusing water molecules with the surrounding tissue micro-structures to create contrast. As such, it is well suited for in-vivo non-invasive white matter structure imaging. Progress in dMRI acquisition, from diffusion tensor imaging (DTI) [1] to high angular resolution diffusion imaging (HARDI), allowed application of more insightful mathematical tools in dMRI analysis. As a consequence, a number of biophysically inspired models which make finer link between underlying tissue microstructures and observed signals have been proposed [23/41516. Several studies have shown that the parameters of these models can be used in the evaluation of several neurological diseases 67789. However, non-linear optimizers employed for the models' parameter estimation, such as Markov chain Monte Carlo (MCMC) [2, Levenberg-Marquardt [34, Gauss-Newton [5], exhibit high computational complexity and often require high number of acquisition points 345, which is not clinically applicable.

Given that a single voxel of dMRI volume contains a high dimensional q-space signal, entire brain scan contains hundreds of thousands dMRI signals. This large 
quantity of data opens the door to deep learning (DL) approaches for various problems where voxel-wise estimation is required. In [10, a fully connected neural network (FCN) was proposed for the estimation of diffusion kurtosis and microstructure parameters from dMRI data obtained with a significantly reduced acquisition protocol. For a similar problem, a sparse reconstruction framework has been unfolded into a DL approach in [11] and was further improved by incorporating long-short term memory (LSTM) units in [12. In [13, a model inspired by [11 was developed for single-shell microstructure estimation. Furthermore, DL was used for learning features related to certain neurological disease. In [14] a CNN model adjusted to the dMRI data domain which combines inter- and intra-voxel features, was proposed for classification into Parkinson disease and control group. Rotation and translation equivariant network was developed and successfully applied on the problem of multiple sclerosis lesion segmentation in 15. Furthermore, as dMRI acquisition protocols significantly vary over different research centers and studies, a comparison of harmonization techniques was conducted and showed potential of deep CNN based approaches over parametric models [16. A number of approaches was proposed for the estimation of fiber orientation distribution functions (fODF). In 17/18, estimation of fODFs was achieved with planar CNNs applied on dMRI data in spectral and signal domain. For the same task, spherical U-nets which exhibit rotational equivariance were proposed in [19|20].

Prior to the models tailored to the spherical nature of dMRI [14]15]19]20, a number of rotationally equivariant DL models were proposed for arbitrary $S^{2}$ signals. One of the prominent spherical CNNs was proposed in [21], where convolutions are performed in spectral instead of signal domain in order to avoid computationally expensive interpolations. Since convolution of two $S^{2}$ signals gives a signal in $S O(3)$ manifold, apart from the first layer, in all the following, convolution is performed between $S O(3)$ signals and kernels [21. In the same work and in accordance with planar CNNs, non-linearity is applied in signal domain, which requires computationally demanding transformation from Fourier to $S O(3)$ domain. To address this problem, in 22, a spherical CNN model with zonal kernels was proposed. Another issue that arises from the non-linearity in signal domain is the introduction of high frequency components, which might introduce aliasing. In the work presented by [23], a fully Fourier space CNN was proposed. This model applies non-linearities of quadratic nature in spectral domain, so it completely eliminates conversions from spectral to signal space and the distortions introduced by aliasing.

In this work, we propose a spherical CNN with rotation equivariant Fourier domain convolutional and non-linear layers, where trainable kernels and biases are represented in Fourier domain of $S^{2}$ and $S O(3)$ manifold. Whereas the spherical CNN models proposed in 212223] assume that input is bandlimited $S^{2}$ signal acquired at equiangular grid as one defined in [27, our model is tailored to the spherical dMRI signals acquired at randomly uniformly distributed points 28] and which are affected by significant Rician noise. The proposed model is evaluated on the problem of estimation of neurite orientation dispersion and 
density imaging (NODDI) [5] parameters from the data from the Human Connectome Project (HCP) [29].

\section{Theory}

In this section, we provide theoretical grounds related to $S^{2}$ and $S O(3)$ signals which are used to define our spherical CNN model described in the section Methods. The presented theory is, to a certain extent, common to the multiple rotation equivariant DL models proposed in 21222315.

An arbitrary square integrable $\left(\mathbb{L}^{2}\right)$ bandlimited signal $s: S^{2} \rightarrow \mathbb{C}$ can be represented as a linear combination of spherical harmonic $(\mathrm{SH})$ basis elements as

$$
s(\mathbf{r})=\sum_{l=0}^{B} \sum_{m=-l}^{m=l} \hat{s}_{l}^{m} Y_{l}^{m}(\mathbf{r})
$$

where $\mathbf{r} \in \mathbb{R}^{3},\|\mathbf{r}\|=1 . Y_{l}^{m}: S^{2} \rightarrow \mathbb{C}$ is the SH basis element of degree $l$ and order $m . \hat{s}_{l}^{m}$ is the corresponding SH coefficient and $B$ is signal's bandwidth. As the $\mathrm{SH}$ basis are orthogonal, $\hat{s}_{l}^{m}$ can be obtained as

$$
\hat{s}_{l}^{m}=\int_{S^{2}} s(\mathbf{r}) Y_{l}^{m *}(\mathbf{r}) d \mathbf{r} .
$$

Given two $\mathbb{L}^{2}$ signals $f, g: S^{2} \rightarrow \mathbb{C}$ of bandwidth $B$ their convolution is defined as

$$
[f * g](R)=\int_{S^{2}} f(\mathbf{r}) g^{*}\left(R^{-1} \mathbf{r}\right) d \mathbf{r}=\sum_{l=0}^{B} \sum_{m=-l}^{l} \sum_{n=-l}^{l} D_{l}^{m n}(R) \hat{f}_{l}^{m} \hat{g}_{l}^{n *},
$$

where $R=R(\alpha, \beta, \gamma) \in S O(3), \hat{f}_{l}^{m}$ and $\hat{g}_{l}^{n}$ are SH coefficients of degree $l$ and orders $m$ and $n$ of the signals $f(\mathbf{r})$ and $g(\mathbf{r})$ 24]. $D_{l}^{m n}: S O(3) \rightarrow \mathbb{C}$ is an element of Wigner-D matrix of degree $l$ and orders $m$ and $n$. Consequently, the convolution of two spherical signals results in a signal whose domain is the $S O(3)$ manifold. An arbitrary $\mathbb{L}^{2}$ bandlimited signal $h: S O(3) \rightarrow \mathbb{C}$ can be represented as a linear combination of the elements of Wigner-D matrices (referred here to as rotational harmonics $(\mathrm{RH})$ ) as

$$
h(R)=\sum_{l=0}^{B} \sum_{m=-l}^{m=l} \sum_{n=-l}^{n=l} \hat{H}_{l}^{m n} D_{l}^{m n}(R) .
$$

where $\hat{H}_{l}^{m n}$ is the RH coefficient associated with the element of Wigner-D matrix $D_{l}^{m n}(R)$ and $B$ is signal's bandwidth [24. If $h(R)=[f * g](R)$, then from equations 3 and $4 \hat{H}_{l}=\hat{\mathbf{f}}_{\mathbf{l}} \hat{\mathbf{g}}_{\mathbf{l}}^{H}$ where $\hat{\mathbf{f}}_{\mathbf{l}}$ and $\hat{\mathbf{g}}_{1} \in \mathbb{C}^{2 l+1}$ contain the SH coefficients of degree $l$ of signals $f(\mathbf{r})$ and $g(\mathbf{r}) . \hat{H}_{l} \in \mathbb{C}^{(2 l+1) \times(2 l+1)}$ contains the RH coefficients of degree $l$ of signal $h(R)$. Convolution of two $\mathbb{L}^{2}$ signals $f, g: S O(3) \rightarrow \mathbb{C}$ of 
bandwidth $B, h(R)=[f * g](R)$, is defined as

$h(R)=\int_{S O(3)} f(Q) g^{*}\left(R^{-1} Q\right) d Q=\sum_{l=0}^{B} \frac{8 \pi^{2}}{2 l+1} \sum_{m=-l}^{l} \sum_{n=-l}^{l} D_{l}^{m n}(R) \sum_{k=-l}^{l} \hat{F}_{l}^{m k} \hat{G}_{l}^{n k *}$

where $\hat{F}_{l}^{m k}$ and $\hat{G}_{l}^{k n}$ are the RH coefficients of degree $l$ and orders $m, k$ and $k, n$ of signals $f(R)$ and $g(R)$ 24]. From equations 4 and 5 , we have $\hat{H}_{l}=$ $\frac{8 \pi^{2}}{2 l+1} \hat{F}_{l} \hat{G}_{l}^{H}$ where $\hat{H}_{l}, \hat{F}_{l}, \hat{G}_{l} \in \mathbb{C}^{(2 l+1) \times(2 l+1)}$ contain the RH coefficients of degree $l$ of functions $h(R), f(R)$ and $g(R)$. Product of two $\mathbb{L}^{2}$ signals $f, g: S O(3) \rightarrow \mathbb{C}$ of bandwidths $B_{f}$ and $B_{g}, h(R)=f(R) \times g(R)$, is defined as

$$
h(R)=\sum_{l^{\prime}=0}^{B_{f}} \sum_{m^{\prime}=-l^{\prime}}^{l^{\prime}} \sum_{n^{\prime}=-l^{\prime}}^{l^{\prime}} \sum_{l^{\prime \prime}=0}^{B_{g}} \sum_{m^{\prime \prime}=-l^{\prime \prime}}^{l^{\prime \prime}} \sum_{n^{\prime \prime}=-l^{\prime \prime}}^{l^{\prime \prime}} \hat{F}_{l^{\prime}}^{m^{\prime} n^{\prime}} \hat{G}_{l^{\prime \prime}}^{m^{\prime \prime} n^{\prime \prime}} D_{l^{\prime}}^{m^{\prime} n^{\prime}}(R) D_{l^{\prime \prime}}^{m^{\prime \prime} n^{\prime \prime}}(R)
$$

where product of elements of Wigner-D matrices can be represented as a linear combination of elements of Wigner-D matrices using Clebsch-Gordan coefficients $<l^{\prime} k^{\prime} l^{\prime \prime} k^{\prime \prime} \mid l k>$ which are non-zero only if $k=k^{\prime}+k^{\prime \prime}$ as

$$
D_{l^{\prime}}^{m^{\prime} n^{\prime}}(R) D_{l^{\prime \prime}}^{m^{\prime \prime} n^{\prime \prime}}(R)=\sum_{l=\left|l^{\prime}-l^{\prime \prime}\right|}^{l^{\prime}+l^{\prime \prime}}<l^{\prime} m^{\prime} l^{\prime \prime} m^{\prime \prime}\left|l m><l^{\prime} n^{\prime} l^{\prime \prime} n^{\prime \prime}\right| l n>D_{l}^{m n}(R)
$$

where $m=m^{\prime}+m^{\prime \prime}$ and $n=n^{\prime}+n^{\prime \prime}$ [26. This means that $h(R)$ is an $S O(3)$ signal of bandwidth $B_{g}+B_{f}$ whose $\mathrm{RH}$ coefficients, $\hat{H}_{l}$, in vector-matrix notation can be represented as

$$
\hat{H}_{l}=\sum_{l^{\prime}, l^{\prime \prime}} C_{l^{\prime}, l^{\prime \prime}}^{l}{ }^{T}\left[\hat{F}_{l^{\prime}} \otimes \hat{G}_{l^{\prime \prime}}\right] C_{l^{\prime}, l^{\prime \prime}}^{l} \quad \text { s.t. } \quad\left|l^{\prime}-l^{\prime \prime}\right| \leq l \leq l^{\prime}+l^{\prime \prime}
$$

where matrix $C_{l^{\prime}, l^{\prime \prime}}^{l} \in \mathbb{R}^{\left(2 l^{\prime}+1\right)\left(2 l^{\prime \prime}+1\right) \times(2 l+1)}$ is a sparse matrix containing ClebschGordan coefficients. As we are dealing with real $S^{2}$ signals, we use real $\mathrm{SH}$ and Wigner-D basis and accordingly defined Clebsch-Gordan matrices using the unitary matrices as in 25 .

\section{Methods}

Architecture of the model proposed in this work is illustrated in Figure 1. It is composed of denoising layers, $S^{2}$ and $S O(3)$ convolutional layers, layers with quadratic non-linearity and fully connected layers at the end. From denoised input signals and each feature map, after non-linearity is applied, rotation invariant power spectrum features are extracted. They are concatenated and fed into fully connected layers which perform final inference. 


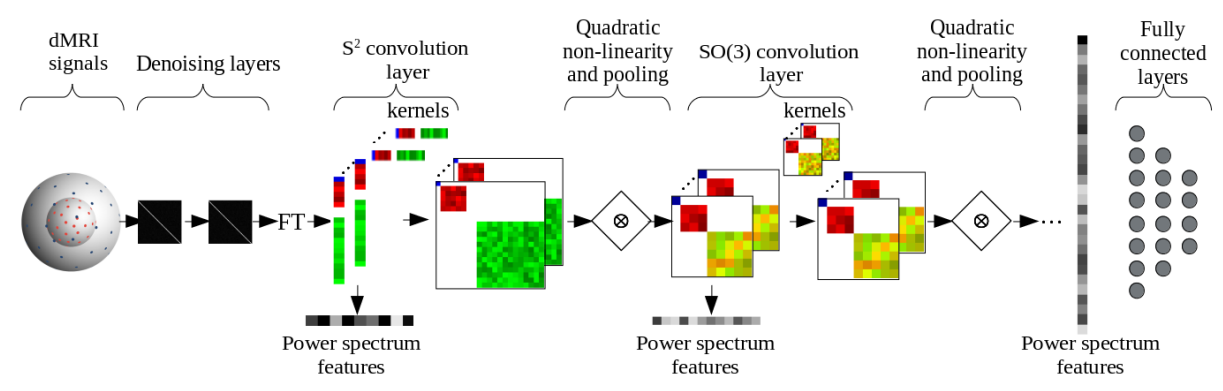

Fig. 1: Illustration of proposed spherical CNN architecture

\subsection{Denoising layers}

Since dMRI signals used in our experiments are acquired at random uniformly distributed points 28] we cannot use quadrature rule for computation of SH coefficients as in 21|22|23]. In addition, the signals are affected by significant Rician noise. As dMRI signals are positive, we define denoising layer as $\operatorname{Re} L U((I+$ $\lambda W) s$ ) where $I$ is identity matrix, $W$ are trainable weights, $s$ is input signal and $\lambda$ is parameter which should ensure that matrix $(I+\lambda W)$ is not too far from identity matrix.

\subsection{Convolutional layers}

Convolutions are performed in spectral domain as firstly proposed in [21. Given input denoised dMRI signal of bandwidth $L$ acquired at $K$ shells (channels) $\left\{s_{k}:\left\{\left(\theta_{i}, \phi_{i}\right)\right\}_{i=1}^{N_{k}} \rightarrow R\right\}_{k=1}^{K}$, SH coefficients $\left\{\left\{\hat{\mathbf{s}}_{l}^{k} \in \mathbb{R}^{2 l+1}\right\}_{l=0}^{L}\right\}_{k=1}^{K}$ are estimated using real SH basis 25], orthonormalized using Gram-Schmidt process. First convolutional layer contains weights and biases expressed in terms of SH coefficients $\left\{\left\{\left\{\hat{\mathbf{w}}_{l}^{k i} \in \mathbb{R}^{2 l+1}\right\}_{l=0}^{L}\right\}_{k=1}^{K}\right\}_{i=1}^{M},\left\{\hat{\mathbf{b}}^{i} \in \mathbb{R}^{1}\right\}_{i=1}^{M}$, where $M$ is the number of output channels. Convolution is given by

$$
\hat{F}_{l}^{i}=\sum_{k=1}^{K} \hat{\mathbf{s}}_{l}^{k} \hat{\mathbf{w}}_{l}^{k i T} \quad \text { if } \quad l \neq 0, \quad \hat{F}_{l}^{i}=\sum_{k=1}^{K} \hat{\mathbf{s}}_{l}^{k} \hat{\mathbf{w}}_{l}^{k i T}+\hat{\mathbf{b}}^{i} \quad \text { if } \quad l=0,
$$

where $\left\{\hat{F}_{l}^{i} \in \mathbb{R}^{(2 l+1) \times(2 l+1)}\right\}_{i=1}^{M}$ are the real $\mathrm{RH}$ coefficients of the resulting $S O(3)$ signals. As in 21, all the following convolutional layers contain convolutions between $S O(3)$ feature maps and kernels. For a layer $n>1$, weights and biases are expressed in terms of real RH coefficients $\left\{\left\{\left\{\hat{W}_{l}^{p q} \in \mathbb{R}^{(2 l+1) \times(2 l+1)}\right\}_{l=0}^{L_{n}}\right\}_{p=1}^{P}\right\}_{q=1}^{Q}$, $\left\{\hat{B}^{q} \in \mathbb{R}^{1}\right\}_{q=1}^{Q}$, where $P$ and $Q$ are the number of input and output channels and $L_{n}$ is bandwidth of input feature maps. Given the RH coefficients of the input feature maps $\left\{\left\{\hat{S}_{l}^{p} \in \mathbb{R}^{(2 l+1) \times(2 l+1)}\right\}_{l=0}^{L_{n}}\right\}_{p=1}^{P}$ convolution is given by

$\hat{F}_{l}^{q}=\frac{8 \pi^{2}}{2 l+1} \sum_{p=1}^{P} \hat{S}_{l}^{p} \hat{W}_{l}^{p q T} \quad$ if $\quad l \neq 0, \quad \hat{F}_{l}^{q}=\frac{8 \pi^{2}}{2 l+1} \sum_{p=1}^{P} \hat{S}_{l}^{p} \hat{W}_{l}^{p q T}+\hat{B}^{q} \quad$ if $\quad l=0$ 
where $\left\{\hat{F}_{l}^{q} \in \mathbb{R}^{(2 l+1) \times(2 l+1)}\right\}_{q=1}^{Q}$ are $\mathrm{RH}$ coefficients of the resulting $S O(3)$ signals.

\subsection{Non-linearity and pooling}

In the domain of rotation equivariant neural networks, non-linear activations of quadratic nature were firstly introduced in [23, where Clebsch-Gordan decomposition is used to decompose tensor product of $S O(3)$ covariant vectors into irreducible fragments. In our work, we propose a non-linearity $f^{2}(R)$ which is rotationally equivariant and allows to preserve $S O(3)$ feature maps. Given real RH coefficients $\left\{\hat{F}_{l}^{q}\right\}_{l=0}^{L_{n}}$ of feature map $f(R)^{q}$, using Eq. 8. RH coefficients of $h(R)^{q}=f(R)^{q} \times f(R)^{q}$ can be expressed as

$$
\hat{H}_{l}^{q}=\sum_{l^{\prime}, l^{\prime \prime}} \mathcal{C}_{l^{\prime}, l^{\prime \prime}}^{l}{ }^{T}\left[\hat{F}_{l^{\prime}}^{q} \otimes \hat{F}_{l^{\prime \prime}}^{q}\right] \mathcal{C}_{l^{\prime}, l^{\prime \prime}}^{l} \quad \text { s.t. } \quad\left|l^{\prime}-l^{\prime \prime}\right| \leq l \leq l^{\prime}+l^{\prime \prime}
$$

where $\mathcal{C}_{l^{\prime}, l^{\prime \prime}}^{l}$ are corresponding real Clebsch-Gordan matrices 25]. Although the bandwidth of the function $h(R)^{q}$ is $2 L_{n}$ to achieve effect of pooling (reducing feature map resolution) $\mathrm{RH}$ coefficients of degree higher then some $L_{m}\left(L_{m} \leq\right.$ $\left.L_{n}\right)$ are discarded as in 2123.

\subsection{Rotation invariant feature vector}

As goal of the cascade of convolutional and non-linear layers is to extract rotation invariant features, in [23] fragments of degree $l=0$ at the output of each nonlinear layer are concatenated (including $l=0$ fragment of input signals) and represent rotation invariant vector that is further fed to a FCN. In our model, $\mathrm{RH}$ coefficients of degree $l=0$ are rotation invariant as well. In addition, from the generalization of Parseval's theorem to $S^{2}$ and $S O(3)$ signals, we can notice that angular and rotation power spectra defined as

$$
P_{f f}(l)=\sum_{m=-l}^{l}\left|\hat{f}_{l}^{m}\right|^{2} \quad \text { and } \quad P_{h h}(l)=\frac{8 \pi^{2}}{2 l+1} \sum_{m=-l}^{l} \sum_{n=-l}^{l}\left|\hat{H}_{l}^{m n}\right|^{2}
$$

where $\hat{f}_{l}^{m}$ and $\hat{H}_{l}^{m n}$ are SH and RH coefficients of arbitrary $\mathbb{L}^{2}$ functions $f$ and $h$ on $S^{2}$ and $S O(3)$, are rotation invariant as well. Given this, we create rotation invariant feature vectors by concatenating the power spectra values of all degrees $l$ of outputs of each non-linear layer and input signals. As in 23, they are fed to a FCN which performs the final inference.

\section{Experiments}

We used in our experiments real data of 50 subjects from Human Connectome Project (HCP) 29], 30 for training, 10 for validation and 10 testing. Scans are composed of three shells with b-values of 1000, 2000 and $3000 \mathrm{~s} / \mathrm{mm}^{2}$, each with 
90 gradient directions and $18 b=0$ images. Scans were previously registered to $\mathrm{T} 1 \mathrm{w}$ images. To select relevant white matter voxels, brain tissue segmentation computed from T1w images using FAST algorithm 31] implemented in mrtrix library 32 was used. NODDI gold standard parameters, namely intra-axonal volume fraction $\nu_{I C}$, isotropic volume fraction $\nu_{i s o}$ and orientation dispersion $O D$, were estimated using brute2fine optimizer from dmipy toolbox on full acquisition scheme, while the methods are compared on significantly downsampled scheme with 30 sampling points per two shells -1000 and $2000 \mathrm{~s} / \mathrm{mm}^{2}$.

We compared our method with brute 2 fine optimizer and two deep learning approaches, a model developed specifically for NODDI parameter estimation MEDN [11] and a FCN which was initially used for microstructure parameter estimation in [10]. Our model is composed of: (1) two denoising layers of size $60 \times 60,(2)$ three convolutional layers of bandwidths 6,4 and 2 with convolutional kernels of sizes $2 \times 8 \times 28,8 \times 16 \times 165,16 \times 32 \times 35$, followed by quadratic non-linearity, and (3) four fully connected layers of sizes $128 \times 128,128 \times 64$, $64 \times 32,32 \times 3$, followed by ReLU, except the last one which is followed by sigmoid activation. FCN model has seven fully connected layers of size $60 \times 256$, $256 \times 192,192 \times 128,128 \times 64,64 \times 32,32 \times 16$ and $16 \times 3$. All models are trained over 200 epochs. In each epoch 12800 dMRI signals were randomly selected from white matter region from each of 10 randomly selected training subjects. Models are trained in a way that they see same amount of data during training. For all three DL approaches, loss function is defined as mean squared error (MSE). For MEDN and FCN initial learning rate is 0.0001 and for our model it is 0.001 , after 50 epochs they are reduced by factor 5 and after 100 by factor 10 of initial values. Batch size is 128. Total number of trainable parameters is 100579,109 865 and 73691 for FCN, MEDN and our model respectively. Codes and trained models are provided at https://gitlab.inria.fr/ssedlar/fourier_s2cnn.

\section{$5 \quad$ Results and conclusions}

We compared results in terms of mean absolute value and its standard deviation over 10 testing subjects. Quantitative comparison of the results is given in Table 1. where we can see that our proposed approach yields lower average errors. In Figure 2, qualitative comparison between methods is provided for one axial slice of a testing subject. Whereas, the differences between brute2fine and DL approaches are noticeable, some differences between DL approaches can be seen in OD estimation, where MEDN and FCN tend to overestimate OD values. Furthermore, to investigate rotation invariance of DL approaches, we have trained models on data whose diffusion tensor fit direction is in range $\left[0, \frac{\pi}{6}\right)\left(\right.$ or $\left.\left(\frac{5 \pi}{6}, \pi\right)\right)$ and the quantitative results of the experiments are provided in Table 2 clearly indicating rotation invariance of our model.

In this work we have proposed a rotation invariant model for signals acquired at randomly distributed points on sphere and affected by considerable noise such as dMRI signals. We have demonstrated that the proposed model can be used successfully in the domain of dMRI analysis such as microstructure 
Table 1: Mean absolute error and std over 10 testing subjects

\begin{tabular}{|c|c|c|c|}
\hline Model & $\nu_{I C}$ & $\nu_{I S O}$ & $O D$ \\
\hline brute2fine & $(5.06 \pm 0.36) \times 10^{-2}$ & $(3.87 \pm 0.24) \times 10^{-2}$ & $(2.97 \pm 0.27) \times 10^{-2}$ \\
\hline FCN & $(3.86 \pm 0.28) \times 10^{-2}$ & $(3.14 \pm 0.21) \times 10^{-2}$ & $(2.26 \pm 0.15) \times 10^{-2}$ \\
\hline MEDN & $(3.81 \pm 0.28) \times 10^{-2}$ & $(3.16 \pm 0.20) \times 10^{-2}$ & $(2.17 \pm 0.16) \times 10^{-2}$ \\
\hline Ours & $(3.77 \pm 0.28) \times 10^{-2}$ & $(3.00 \pm 0.21) \times 10^{-2}$ & $(2.08 \pm 0.16) \times 10^{-2}$ \\
\hline
\end{tabular}

Table 2: Mean absolute error and std over 10 testing subjects with direction restricted training

\begin{tabular}{|c|c|c|c|}
\hline Model & $\nu_{I C}$ & $\nu_{I S O}$ & $O D$ \\
\hline FCN & $(4.73 \pm 0.16) \times 10^{-2}$ & $(3.43 \pm 0.18) \times 10^{-2}$ & $(18.31 \pm 0.77) \times 10^{-2}$ \\
\hline MEDN & $(5.09 \pm 0.21) \times 10^{-2}$ & $(3.76 \pm 0.19) \times 10^{-2}$ & $(13.46 \pm 0.50) \times 10^{-2}$ \\
\hline Ours & $(3.99 \pm 0.31) \times 10^{-2}$ & $(3.19 \pm 0.20) \times 10^{-2}$ & $(4.10 \pm 0.15) \times 10^{-2}$ \\
\hline
\end{tabular}

parameter estimation. In the future work we will investigate how computational complexity of quadratic activation function can be decreased and how the model performance can be improved by incorporating neighbourhood information.

\section{Acknowledgment}

This work was supported by the ERC under the European Union's Horizon 2020 research and innovation program (ERC Advanced Grant agreement No 694665:CoBCoM : Computational Brain Connectivity Mapping).

This work has been partly supported by the French government, through the 3IA Côte d'Azur Investments in the Future project managed by the National Research Agency (ANR) with the reference number ANR-19-P3IA-0002.

Data were provided [in part] by the Human Connectome Project, WU-Minn Consortium (Principal Investigators: David Van Essen and Kamil Ugurbil; 1U54MH091657) funded by the 16 NIH Institutes and Centers that support the NIH Blueprint for Neuroscience Research; and by the McDonnell Center for Systems Neuroscience at Washington University.

The authors are grateful to Inria Sophia Antipolis - Méditerranée https:// wiki.inria.fr/ClustersSophia/Usage_policy 'Nef' computation cluster for providing resources and support.

The authors are grateful to the OPAL infrastructure from Université Côte d'Azur for providing resources and support.

\section{References}

1. Le Bihan, Denis, et al. "Diffusion tensor imaging: concepts and applications." Journal of Magnetic Resonance Imaging: An Official Journal of the International Society for Magnetic Resonance in Medicine 13.4 (2001): 534-546. 


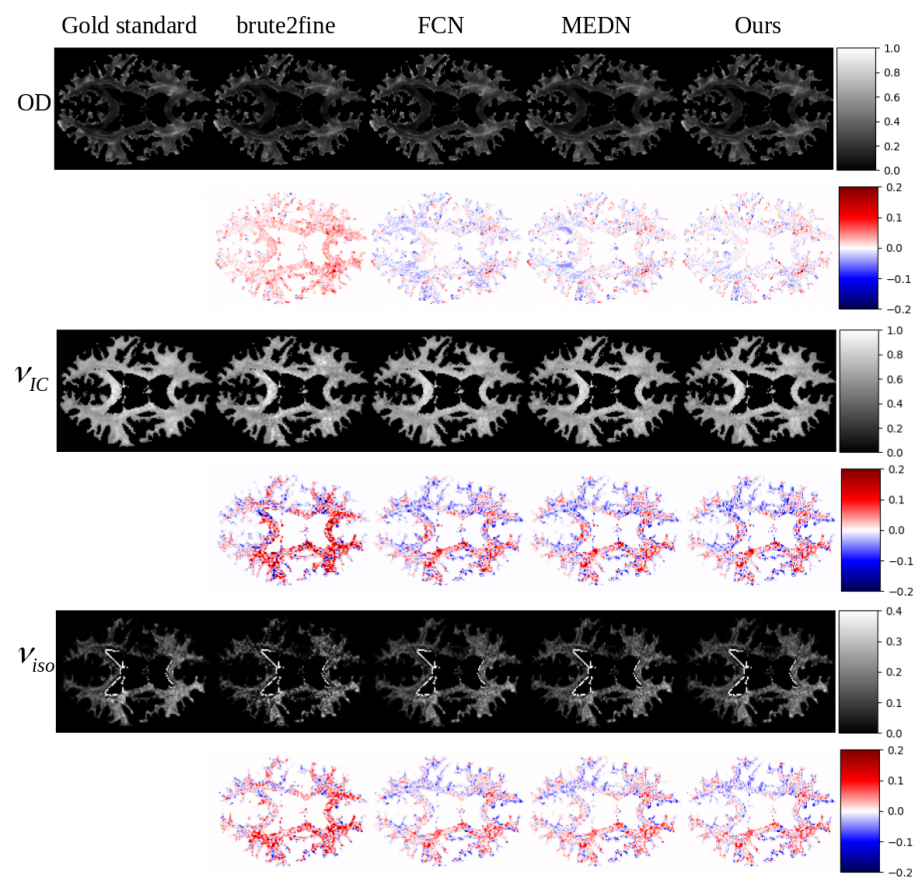

Fig. 2: Qualitative comparison of NODDI parameter estimation and differences with respect to gold standard. Red color indicates underestimation and blue color overestimation.

2. Behrens, Timothy EJ, et al. "Characterization and propagation of uncertainty in diffusion-weighted MR imaging." Magnetic Resonance in Medicine: An Official Journal of the International Society for Magnetic Resonance in Medicine 50.5 (2003): 1077-1088.

3. Assaf, Yaniv, and Peter J. Basser. "Composite hindered and restricted model of diffusion (CHARMED) MR imaging of the human brain." Neuroimage 27.1 (2005): 48-58.

4. Assaf, Yaniv, et al. "AxCaliber: a method for measuring axon diameter distribution from diffusion MRI." Magnetic Resonance in Medicine: An Official Journal of the International Society for Magnetic Resonance in Medicine 59.6 (2008): 1347-1354.

5. Zhang, Hui, et al. "NODDI: practical in vivo neurite orientation dispersion and density imaging of the human brain." Neuroimage 61.4 (2012): 1000-1016.

6. Panagiotaki, Eletheria, et al. "Noninvasive quantification of solid tumor microstructure using VERDICT MRI." Cancer research 74.7 (2014): 1902-1912.

7. De Santis, Silvia, et al. "Early axonal damage in normal appearing white matter in multiple sclerosis: Novel insights from multi-shell diffusion MRI." 2017 39th Annual International Conference of the IEEE Engineering in Medicine and Biology Society (EMBC). IEEE, 2017.

8. Schneider, Torben, et al. "Sensitivity of multi-shell NODDI to multiple sclerosis white matter changes: a pilot study." Functional neurology 32.2 (2017): 97. 
9. Broad, Rebecca J., et al. "Neurite orientation and dispersion density imaging (NODDI) detects cortical and corticospinal tract degeneration in ALS." Journal of Neurology, Neurosurgery Psychiatry 90.4 (2019): 404-411.

10. Golkov, Vladimir, et al. "Q-space deep learning: twelve-fold shorter and model-free diffusion MRI scans." IEEE transactions on medical imaging 35.5 (2016): 1344-1351.

11. Ye, Chuyang. "Estimation of tissue microstructure using a deep network inspired by a sparse reconstruction framework." International Conference on Information Processing in Medical Imaging. Springer, Cham, 2017.

12. Ye, Chuyang, Xiuli Li, and Jingnan Chen. "A deep network for tissue microstructure estimation using modified LSTM units." Medical image analysis 55 (2019): 49-64.

13. Faiyaz, Abrar, et al. "DLpN: Single-Shell NODDI Using Deep Learner Estimated Isotropic Volume Fraction." arXiv preprint arXiv:2102.02772 (2021).

14. Banerjee, Monami, et al. "Dmr-cnn: A cnn tailored for dmr scans with applications to pd classification." 2019 IEEE 16th International Symposium on Biomedical Imaging (ISBI 2019). IEEE, 2019.

15. Müller, Philip, et al. "Rotation-Equivariant Deep Learning for Diffusion MRI." arXiv preprint arXiv:2102.06942 (2021).

16. Ning, Lipeng, et al. "Muti-shell diffusion MRI harmonisation and enhancement challenge (MUSHAC): progress and results." International Conference on Medical Image Computing and Computer-Assisted Intervention. Springer, Cham, 2019.

17. Lin, Zhichao, et al. "Fast learning of fiber orientation distribution function for MR tractography using convolutional neural network." Medical physics 46.7 (2019): 3101-3116.

18. Koppers, Simon, and Dorit Merhof. "Direct estimation of fiber orientations using deep learning in diffusion imaging." International Workshop on Machine Learning in Medical Imaging. Springer, Cham, 2016.

19. Sedlar, Sara, et al. "Diffusion MRI fiber orientation distribution function estimation using voxel-wise spherical U-net." Computational Diffusion MRI, MICCAI Workshop. 2020.

20. Elaldi, Axel, et al. "Equivariant Spherical Deconvolution: Learning Sparse Orientation Distribution Functions from Spherical Data." arXiv preprint arXiv:2102.09462 (2021).

21. Taco S. Cohen, Mario Geiger, Jonas Köhler, Max Welling, Spherical CNNs. International Conference on Learning Representations (ICLR), 2018.

22. Esteves, Carlos, et al. "Learning so (3) equivariant representations with spherical cnns." Proceedings of the European Conference on Computer Vision (ECCV). 2018.

23. Kondor, Risi, Zhen Lin, and Shubhendu Trivedi. "Clebsch-gordan nets: a fully fourier space spherical convolutional neural network." Advances in Neural Information Processing Systems 31 (2018): 10117-10126.

24. Sugiura, M. (1990). Unitary representations and harmonic analysis: an introduction. Elsevier.

25. Homeier, Herbert HH, and E. Otto Steinborn. "Some properties of the coupling coefficients of real spherical harmonics and their relation to Gaunt coefficients." Journal of Molecular Structure: THEOCHEM 368 (1996): 31-37.

26. Rose, Morris Edgar. Elementary theory of angular momentum. Courier Corporation, 1995

27. Driscoll, James R., and Dennis M. Healy. "Computing Fourier transforms and convolutions on the 2-sphere." Advances in applied mathematics 15.2 (1994): 202250 . 
28. Caruyer, Emmanuel, et al. "Design of multishell sampling schemes with uniform coverage in diffusion MRI." Magnetic resonance in medicine 69.6 (2013): 1534-1540.

29. Van Essen, David C., et al. "The WU-Minn human connectome project: an overview." Neuroimage 80 (2013): 62-79.

30. Fick, Rutger HJ, Demian Wassermann, and Rachid Deriche. "The dmipy toolbox: Diffusion mri multi-compartment modeling and microstructure recovery made easy." Frontiers in neuroinformatics 13 (2019): 64.

31. Zhang, Yongyue, Michael Brady, and Stephen Smith. "Segmentation of brain MR images through a hidden Markov random field model and the expectationmaximization algorithm." IEEE transactions on medical imaging 20.1 (2001): 45-57. 32. Tournier, J-Donald, et al. "MRtrix3: A fast, flexible and open software framework for medical image processing and visualisation." NeuroImage 202 (2019): 116137. 